\title{
Nova V5116 Sagittarii and searching for superhumps in nova remnants
}

\author{
A. Dobrotka ${ }^{1}$, A. Retter ${ }^{2}$, and A. Liu ${ }^{3}$ \\ 1 Departement of Physics, Faculty of Materials Science and Technology, Slovak University of Technology in Bratislava, \\ Jána Bottu 25, 91774 Trnava, The Slovak Republic \\ e-mail: andrej.dobrotka@stuba.sk \\ 2 Departement of Astronomy and Astrophysics, Penn State University, 525 Davey Lab, University Park, PA, 16802-6305, USA \\ e-mail: aretter@walla.com \\ 3 Norcape Observatory, PO Box 300, Exmounth, 6707, Australia \\ e-mail: asliu@bigpond.net. au
}

Received 15 May 2007 / Accepted 29 October 2007

ABSTRACT

\begin{abstract}
Aims. We present the period analysis of unfiltered photometric observations of V5116 Sgr (Nova Sgr 2005 \#2) and search for superhump candidates in novae remnants.

Methods. The PDM method for period analysis was used. The masses of the novae componets we are estimated from the secondary mass - orbital period and primary mass - decline time relations.

Results. We find that 13 nights of V5116 Sgr observations in the year 2006 are modulated with a period of $0.1238 \pm 0.0001 \mathrm{~d}$ $(2.9712 \pm 0.0024 \mathrm{~h})$. Following the shape of the phased light curves and no apparent change in the value of the periodicity in different subsamples of the data, we interpreted the period as orbital in nature. The binary system then falls within the period gap of the orbital period distribution of cataclysmic variables. From the maximum magnitude - rate of decline relation, we estimated a maximum absolute visual magnitude of $M_{\mathrm{V} \max }=-8.85 \pm 0.04$ mag using the measured value of decline $t_{2}=6.5 \pm 1.0 \mathrm{~d}$. The mass-period relation for cataclysmic variables yields a secondary mass estimate of about $0.26 \pm 0.05 M_{\odot}$. We propose that V5116 Sgr is a high inclination system showing an irradiation effect of the secondary star. No fully developed accretion disc up to the tidal radius with the value lower than $3.5 \times 10^{10} \mathrm{~cm}$ is probable. The mass ratio was estimated in a few novae and the presence or absence of superhumps in these systems was compared with the mass ratio limit for superhumps of about 0.35 . We find that, in the majority of novae with expected superhumps, this variability has not been found yet. Therefore, more observations of these systems is encouraged.
\end{abstract}

Key words. stars: novae, cataclysmic variables - stars: individual: V5116 Sgr - accretion, accretion disks

\section{Introduction}

Novae are a subclass of cataclysmic variable stars. In these interracting binaries, the white dwarf is accreting the matter transferred from the secondary star. The accretion disc may be formed in the non magnetic case. The intermediate polar systems have a truncated disc and polar systems have magnetic field strong enough to prevent disc formation (see Warner 1995, for a review). The accumulation of a critical amount of accreted material onto the white dwarf surface results in a nova explosion. The distribution of orbital periods in cataclysmic variables shows a period gap between about 2 and $3 \mathrm{~h}$, but novae do not show this lack of objects in the mentioned interval.

The source V5116 Sgr (Nova Sgr 2005 \#2) was discovered by Liller (2005) on 2005 July 4.049 UT. The nova had a magnitude $\sim 8.0$ on two red photographs. An unfiltered CCD image from 2005 July 5.085 UT showed the object at mag 7.2. The spectrum from 2005 July 5.099 UT shows $\mathrm{H}_{\alpha}$ with the FWHM of $\sim 970 \mathrm{~km} \mathrm{~s}^{-1}$. The expansion velocity derived from the sharp P Cyg profile was $\sim 1300 \mathrm{~km} \mathrm{~s}^{-1}$. The position of the nova was measured by Gilmore \& Kilmartin (2005) and Jacques (2005). Gilmore \& Kilmartin (2005) searched for the nova precursor, but no convincing candidate has been found. Russell et al. (2005) performed a $0.8-2.5 \mu \mathrm{m}$ spectroscopy of the nova on 2005 July 15. The object showed emission lines of H I, He I, C I, N I, Ca II, and $\mathrm{O}$ I with an $F W H M \sim 2200 \mathrm{~km} \mathrm{~s}^{-1}$, and He I showed a P Cyg profile at 1.0830 and $2.0581 \mu \mathrm{m}$. No thermal dust emission was observed.

After the nova explosion, the accretions disc is destroyed. The new disc is reformed by the stream of matter flowing from the secondary, interracting with itself and forming a ring in the circularisation radius. The disc starts to form by the viscous shearing. Matter losing angular momentum is moving inward and the excess of angular momentum is transported by the matter flowing outward. The inward moving matter touches the white dwarf and the disc is reformed (Pringle 1981). If the white dwarf is magnetic, the matter interacts with the magnetosphere and is then conducted by the magnetic field to the magnetic poles. The interaction of the flow with the poles of the rotating star is observed as a periodic signal with the spin period of the white dwarf. In the case of intermediate polars, the spin period is usually much shorter than the orbital period (Patterson 1994; Hellier 1996). The polar systems without a disc are synchronous rotators, so the spin period is equal or close to the orbital period (see e.g. Schmidt \& Stockman 1991). Nova V1500 Cyg (polar system without a disc) changed the period from $0.141 \mathrm{~d}$ to $0.138 \mathrm{~d}$ and then stabilised at $0.140 \mathrm{~d}$ (Patterson 1978, 1979a). The difference of $1.8 \%$ between the rotation period of the white dwarf and the orbital period is ascribed to the effects of the nova explosion in 1975 . The synchronisation can be then corrupted by the 
nova explosion, but the spin period remain still very close to the orbital period. This is in contrast with intermediate polars with disc presence.

Searching for periods in novae allows study of the orbital distrubution and evolution of these systems. Currently, there are about 50 novae with known orbital periods (Warner 2002) with typical values ranging from 2 to $9 \mathrm{~h}$. The existence of the accretion disc or its renovation after the nova explosion is confirmed by the detection of the superhump period (see e.g. Retter et al. 1997; Kang et al. 2006a) or the spin period of a magnetic white dwarf in the case of intermediate polars (see e.g. Retter et al. 1998).

Superhumps are caused by a precessing accretion disc generally in systems with mass ratio $M_{2} / M_{1}<0.35 \pm 0.02$ (see Patterson et al. 2005, for review), in which the disc radius reaches the 3:1 resonance. The mass ratio indicates that systems with massive primaries and low-mass secondaries are probable superhumpers. Novae in general possess high mass white dwarfs (see e.g. Warner 1995; Smith \& Dhillon 1998; Webbink 1990) and short orbital periods sugest low mass secondaries. It is therefore meaningfull to search for superhump variability in novae with short orbital period.

We have an ongoing program with small telescopes to observe novae and to search for periodicities in their optical light curves. In this paper we report detection of one periodicity in our photometric data $(P=0.1238 \pm 0.0001 \mathrm{~d})$ of V5116 Sgr and discuss the presence of superhumps in nova remnants. In Sect. 2 we present our observational material. The long-term light curve with the period analysis of the data is presented in Sect. 3 and in Sect. 4 we discuss the long-term light-curve behaviour (Sect. 4.1), the results of the period analysis (Sect. 4.2), and superhump search in nova remnants (Sect. 4.3).

\section{Observations}

V5116 Sgr was observed during 13 nights in 2006. The observations included $45.2 \mathrm{~h}$ and 1256 data points in total. The list of observations is presented in Table 1 . The photometry was carried out with a $0.3-\mathrm{m} \mathrm{f} / 6.3$ telescope coupled to an SBIG ST7E CCD camera. The telescope is located in Exmounth, Western Australia. The pixel size of the CCD is $9 \times 9$ microns. This camera is attached to an Optec $\mathrm{f} 5$ focal reducer giving an image field of view of $15 \times 10 \mathrm{arcmin}$. The range of seeing for the data was 2.5-3 arcsec. The exposure times were between 30 and $90 \mathrm{~s} \mathrm{ev-}$ ery $120 \mathrm{~s}$, and no filter was used. Aperture photometry was used in the reduction, with an aperture size of 12 pixels (radius). As comparison star, we used GSC 7392856 with $V=12$ mag and as check star GSC 7392292 with $V=11.6$ mag. The instrumental magnitudes of the stars were derived from the SBIG CCDOPS software and the magnitudes of the comparison and check stars from the GSC.

Figure 1 displays our light curve and the long-term light curve of the nova from the American Association of Variable Star Observers (AAVSO). In Fig. 2 we show the 4 best examples of our observational runs with the sinusoidal fit (with first harmonic) to the data using the period derived in this paper. These variations are seen clearly.

\section{Data analysis}

\subsection{Long-term light curve}

By using the maximum visual magnitude of $V=7.15$, we measured the time required for a decline of two magnitudes from
Table 1. The observational log.

\begin{tabular}{lcccr}
\hline \hline Run & Date & HJD (start) & $\begin{array}{c}t \\
{[\mathrm{~h}]}\end{array}$ & \multicolumn{1}{c}{$N$} \\
\hline 1 & 2006 August 30 & 977.9654 & 4.8 & 133 \\
2 & 2006 August 31 & 978.9766 & 4.5 & 123 \\
3 & 2006 September 21 & 999.9753 & 3.4 & 94 \\
4 & 2006 September 22 & 1000.9831 & 3.3 & 93 \\
5 & 2006 September 24 & 1002.9704 & 3.6 & 101 \\
6 & 2006 September 27 & 1005.9734 & 3.5 & 93 \\
7 & 2006 September 28 & 1006.9792 & 3.3 & 90 \\
8 & 2006 September 29 & 1007.977 & 3.4 & 98 \\
9 & 2006 October 04 & 1012.974 & 3.4 & 94 \\
10 & 2006 October 08 & 1016.9812 & 3.2 & 90 \\
11 & 2006 October 10 & 1018.9811 & 3.0 & 82 \\
12 & 2006 October 15 & 1023.9679 & 3.2 & 91 \\
13 & 2006 October 18 & 1026.9677 & 2.6 & 74 \\
\hline
\end{tabular}

Note: HJD is -2453000 days, $t$ is the duration of the observation in hours and $N$ is the number of frames.

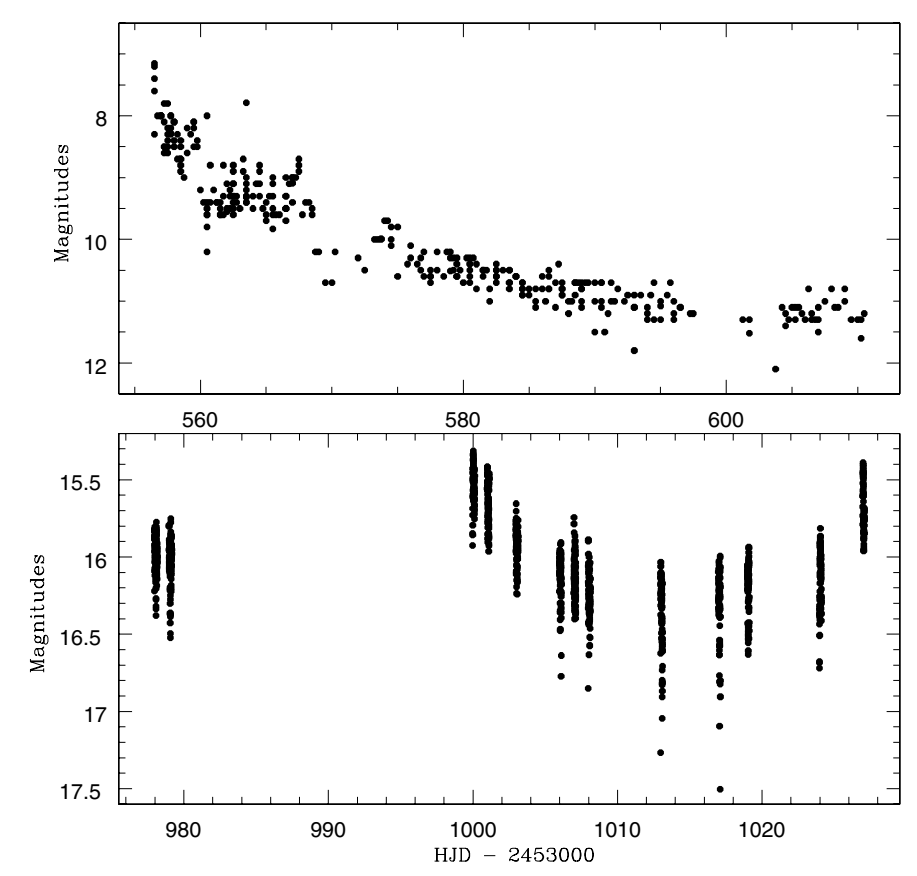

Fig. 1. The AAVSO light curve (upper panel) and the unfiltered light curve of our data (bottom panel).

maximum $t_{2}=6.5 \pm 1.0 \mathrm{~d}$ and three magnitudes from maximum $t_{3}=20.2 \pm 1.9 \mathrm{~d}$ (Fig. $1-$ upper panel). This makes V5116 Sgr a very fast nova according to the classification given in Table 5.4 of Warner (1995). We calculated the visual absolute magnitude at maximum brightness using Eq. (2) of Della Valle \& Livio (1995) and obtained $M_{V \max }=-8.85 \pm 0.04 \mathrm{mag}$.

The bottom panel of the Fig. 1 shows our unfiltered photometry for the nova. The long-term variations with a time scale $\sim 30-40$ days with amplitude $\sim 1$ mag $\sim 450$ days after the outburst is clear. Similar large-scale quasi-periodic brightness oscillations with amplitude 1-1.5 mag months after the maximum were observed, for example, in GK Per, V603 aql, DK Lac (see e.g. Warner 1995) or V4745 Sgr (Csák et al. 2005), with time scales of 5, 12, 25, and $\sim 20$ days, respectively. 

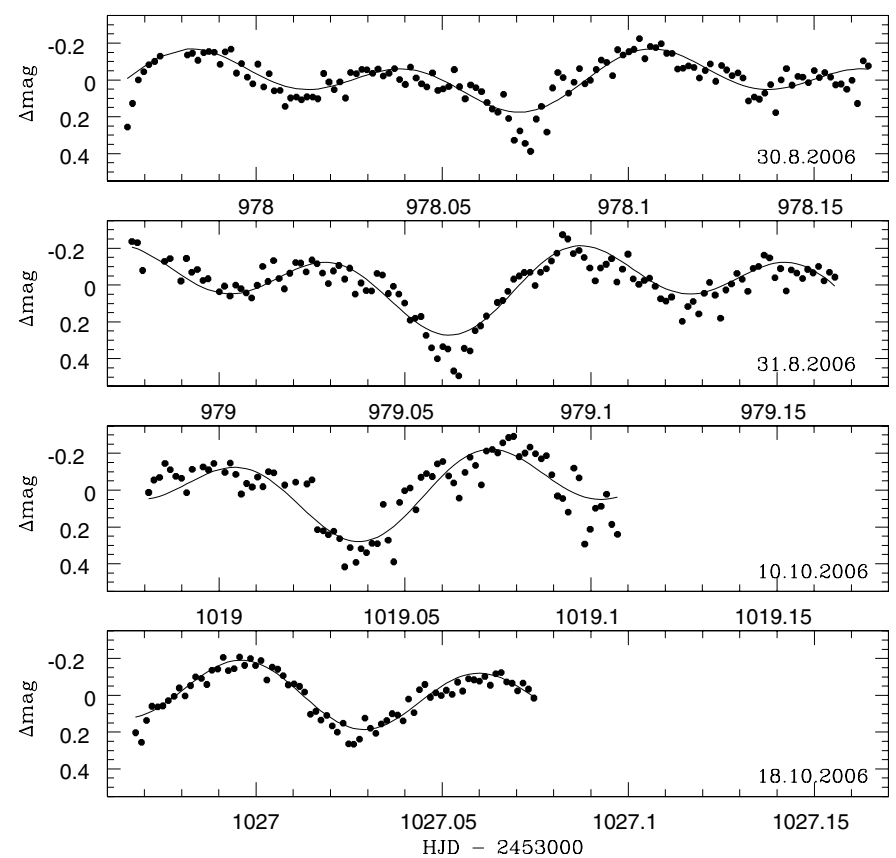

Fig. 2. A selection of our observations (4 detrended best runs; 1, 2, 11, and 13). The solid curve is the sinusoidal fit (plus first harmonic) to the data using the period derived in this paper.

\subsection{Period analysis}

For the period analysis we used the PDM method (Stellingwerf 1978). First, the data were detrended by substracting the linear fit from each night. A simple visual inspection of the runs suggests that the data are modulated with a periodicity of $\sim 1.5 \mathrm{~h}$. This value was then identified as the first harmonic of the real periodicity, making the exact value of the frequency $f=8.080 \pm 0.006 \mathrm{~d}^{-1}$, which corresponds to the periodicity $P=0.1238 \pm 0.0001 \mathrm{~d}(2.9712 \pm 0.0024 \mathrm{~h})$. We calculated the errors in the frequency from the half width at the half minimum of the peaks in the PDM power spectrum.

The results from the period analysis of all the data are depicted in Fig. 3. The suspected periodicity $(f=8.080 \pm$ $\left.0.006 \mathrm{~d}^{-1}, P=0.1238 \pm 0.0001 \mathrm{~d}\right)$ is seen with its $1-\mathrm{d}$ aliases, and the dominant peak is the first harmonic $(2 \times f=$ $\left.16.160 \pm 0.006 \mathrm{~d}^{-1}\right)$. For testing which value is the real signal and which the alias, we performed an $\mathrm{O}-\mathrm{C}$ diagram with a linear fit to the data (Fig. 4; upper panel - O-C for the frequency $8.08 \mathrm{~d}^{-1}$ and lower panel - O-C for the frequency $9.08 \mathrm{~d}^{-1}$ ). The lower panel shows a larger scatter of the $\mathrm{O}-\mathrm{C}$ values compared with the upper panel. This indicates that the real signal is $f=8.080 \pm 0.006 \mathrm{~d}^{-1}$ and the dominant peak at $\sim 16 \mathrm{~d}^{-1}$ is the first harmonic of the real signal.

To test the reality of the peaks, we performed a power spectrum of the synthetic data using a sinusoids with the derived periods (including fundamental $f$, first harmonic $2 \times f$ and $4 \times f$ described later) and phases with amplitudes found from the fits to the data sampled as the original data with introduced Gaussian noise (Fig. 3e). The similarity with the original periodogram is clear. Furthermore, we performed a period analysis of two different subsamples of the data (first subsample - runs 3-8; second subsample - runs 9-13) depicted in Fig. 5. The frequency is present together with its first harmonic in both datasets. The values are $8.090 \pm 0.015 \mathrm{~d}^{-1}$ and $8.080 \pm 0.028 \mathrm{~d}^{-1}$ (first and second subsample, respectively), which is within the errors in both

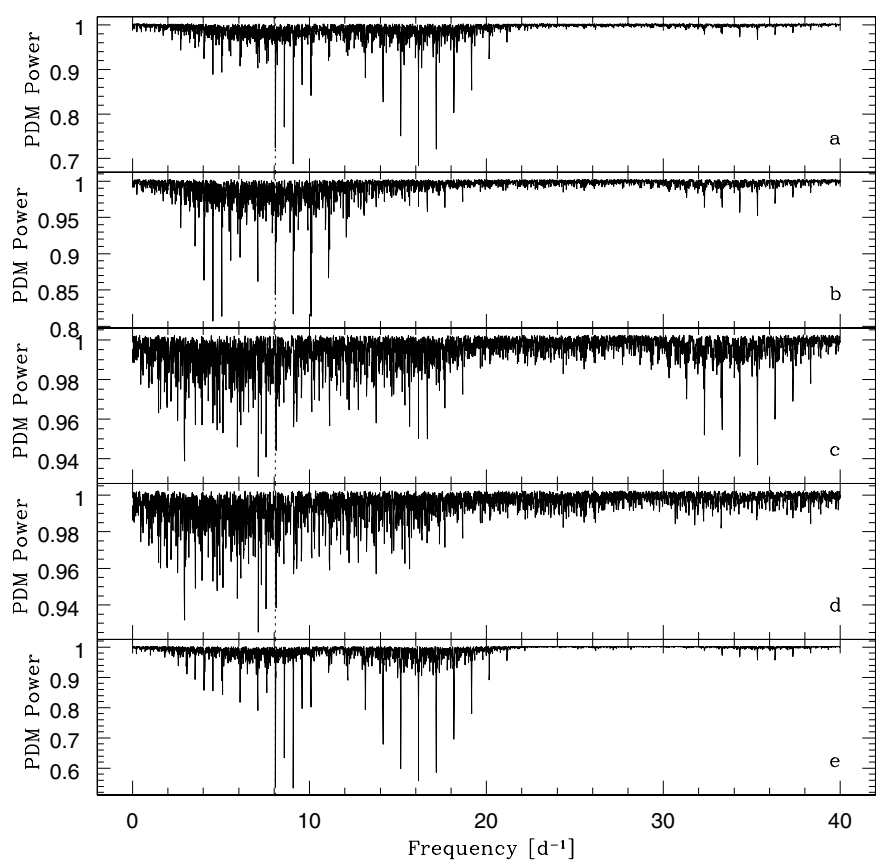

Fig. 3. Power spectra of all the data. Panel a) - raw detrended data (night means substracted); panel b) - data after the substraction of the harmonic frequency $2 \times f$; panel c) - data after the substraction of the harmonic $2 \times f$ and the fundamental frequency $f$; panel d) - data after the substraction of the harmonic $2 \times f$, the fundamental frequency $f$, and the harmonic frequency $4 \times f$; panel e) - synthetic spectrum with the frequencies $f, 2 \times f$, and $4 \times f$. The dashed line represent $f=8.08 \mathrm{~d}^{-1}$.

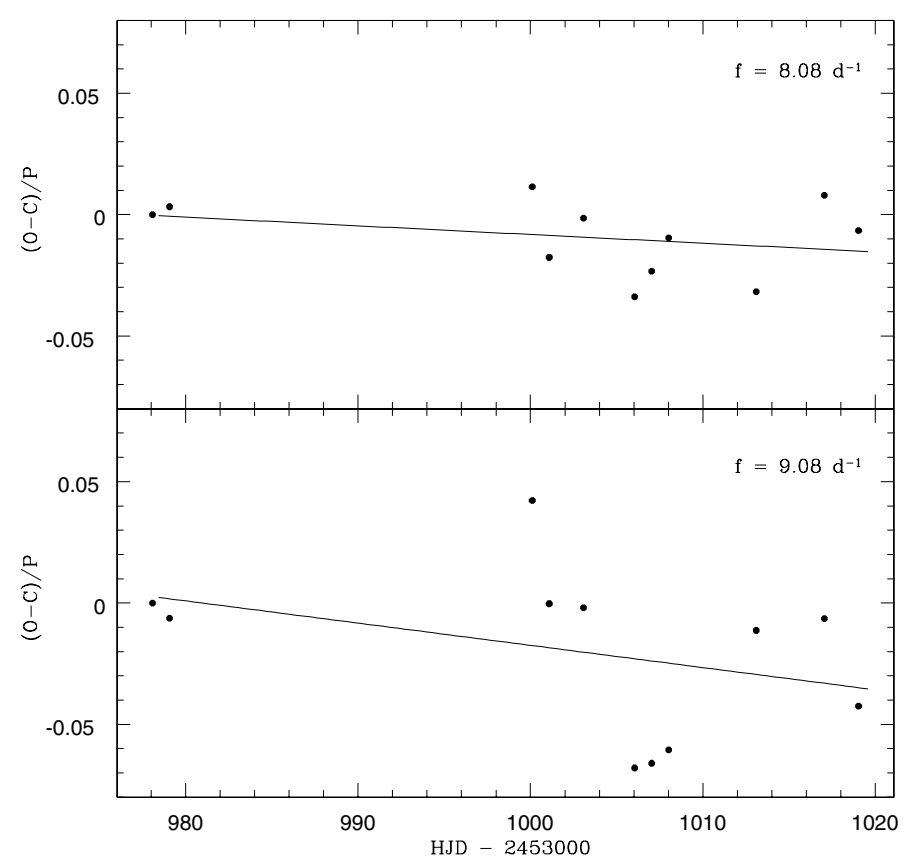

Fig. 4. O-C diagram. Upper panel - for frequency $8.080 \pm 0.006 \mathrm{~d}^{-1}$ $(P=0.1238 \pm 0.0001 \mathrm{~d})$ and lower panel - for frequency $9.080 \pm$ $0.006 \mathrm{~d}^{-1}(P=0.1101 \pm 0.0001 \mathrm{~d})$. The solid line is the linear fit to the data.

cases. We also performed an analysis of the check star and did not identify any periodicity associated with its data.

There is a strong peak near the value $\sim 8.58 \mathrm{~d}^{-1}$. This signal is not real as seen in Figs. 3b,c. After the substraction of $2 \times f$ 


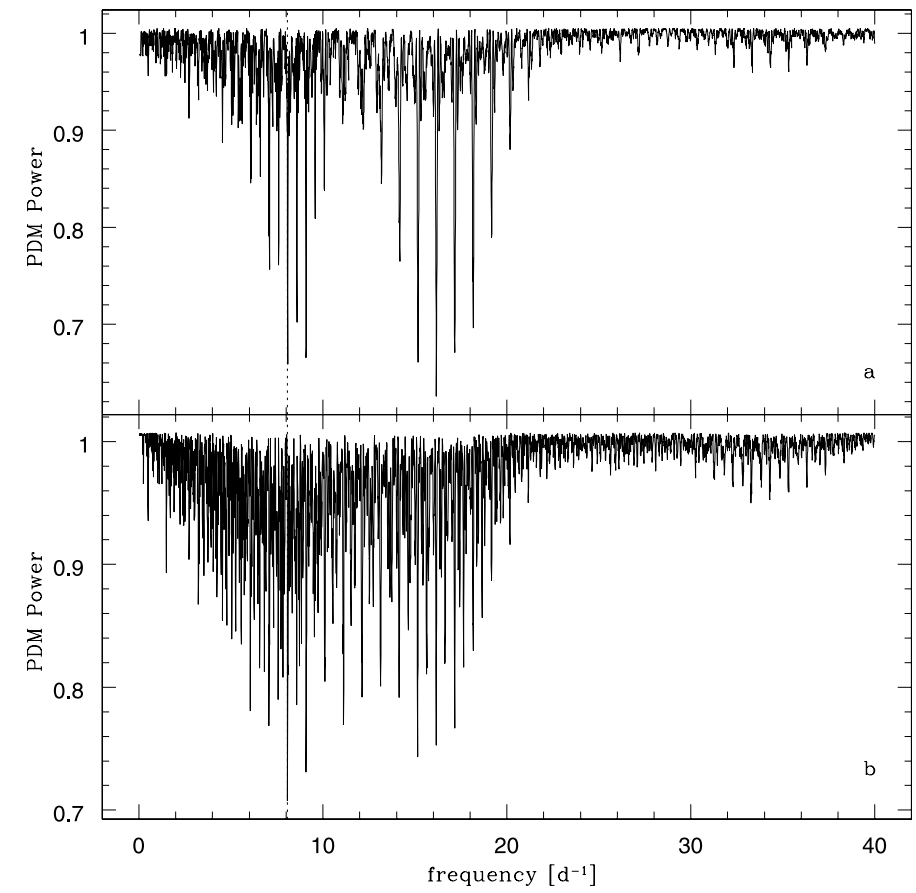

Fig. 5. Power spectra of two different subsamples of the data. Upper panel - runs 3-8; lower panel - runs 9-13. The dashed line represents $f=8.08 \mathrm{~d}^{-1}$.

(Fig. 3b), this peak disappeared, and after a further substraction of $f$ (Fig. 3c), no peak is dominant in this frequency region. This feature also appears in the synthetic power spectrum (Fig. 3e), so it is definitely an alias of $f$.

Another strong feature is a peak at $\sim 35 \mathrm{~d}^{-1}$. Substracting the harmonic frequency $4 \times f$ from the data, after the substraction described in the previous paragraph, this signal dissapared (Fig. 3d). It is another artefact of the real signal $f$. No other dominant peaks are visible with frequencies higher than $40 \mathrm{~d}^{-1}$. No other frequency was then found.

\subsection{The structure of the periodicity}

In Fig. 6 we show the light curve of V5116 Sgr folded on the $0.1238 \mathrm{~d}$ period. The points are averaged magnitudes in 50 equal bins that cover the $0-1$ phase interval. The full amplitude of the mean variation is $0.43 \pm 0.02 \mathrm{mag}$ for primary minimum and $0.30 \pm 0.02 \mathrm{mag}$ for secondary minimum. Using the sinusoidal fit to determine the amplitude is inadequate, because of the non sinusoidal shape of the light curve, we therefore estimated the amplitude by measuring the difference between extrema. The bars are the errors of the mean value. The light curves folded on the periodicity found show typical features of an eclipsing system.

We determided the epoch of minima by fitting low-order polynomials to selected parts of the light curve. The best-fitted (using sinusoidal fits) ephemeris of the periodicity is

$T_{(\min )}(\mathrm{HJD})=2453978.0726+0.1238 E \pm 0.0009 \pm 0.0001$.

\section{Discussion}

\subsection{Long-term variations of V5116 Sgr}

The long-term light curve of the nova V5116 Sgr (Fig. 1 - bottom panel) shows a transition from smooth decline to probable

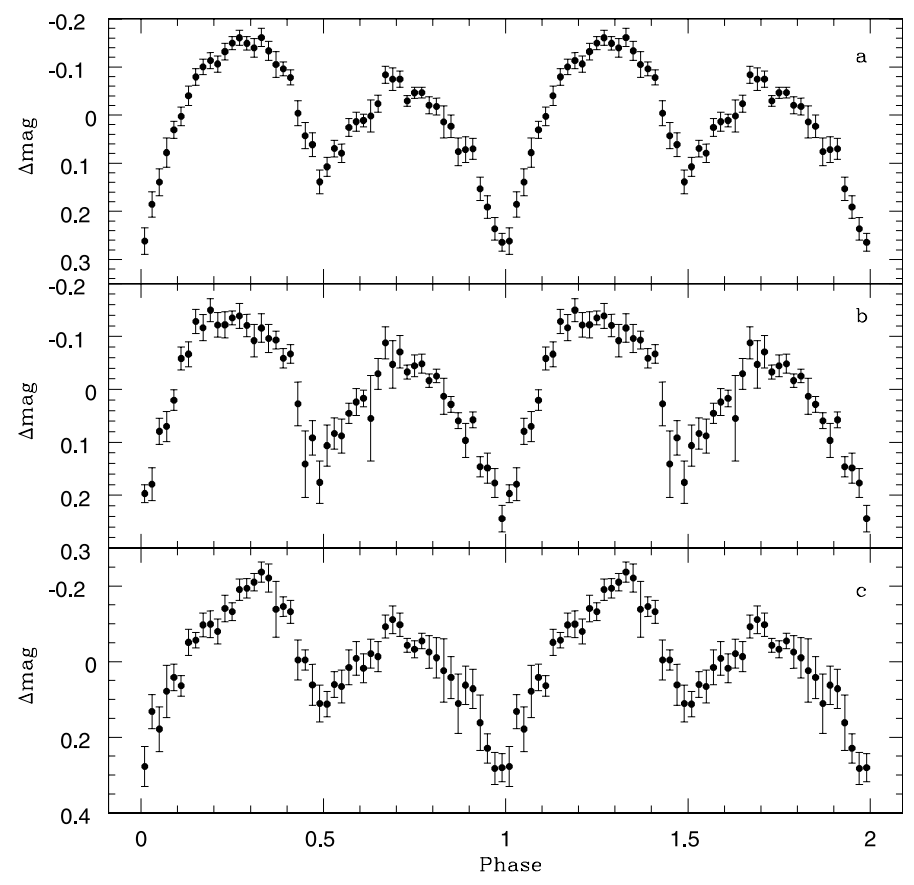

Fig. 6. The light curve of V5116 Sgr folded on the $0.1238 \mathrm{~d}$ period and binned into 50 equal bins. Panel a) - all the data; panel b) - runs 3-8; panel c) - runs 9-13.

oscillations. Several models have been suggested for this "transient" phase. One of them explains the transition as the time when the accretion disc is re-established (Retter 2002). The author proposes a connection between this phase and intermediate polars. There is no indication of the magnetic nature of the white dwarf in V5116 Sgr yet. A spin period of the white dwarf indicating the intermediate polar type of this "transient" phase in novae was detected, for example, in V4745 Sgr by optical observations (Dobrotka et al. 2006a), V1494 Aql (Drake et al. 2003), and V4743 Sgr by X-ray observations (Ness et al. 2003). If the "transient" phase interpretation of Retter (2002) is applicable to V5116 Sgr, the accretion must be then re-established and the accretion disc should be present.

\subsection{Short-term variations of V5116 Sgr}

We have identified one periodicity in the light curve of the nova V5116 Sgr about 15 months after the maximum brightness. Its value is $P=0.1238 \pm 0.0001 \mathrm{~d}(2.9712 \pm 0.0024 \mathrm{~h})$. The upper limits of the period difference between two subset of data with $\simeq 16$ days of mean distance analysed in Fig. 5 is $1.5 \times 10^{-4}$ d, which gives $|\dot{P}| \simeq 0.94 \times 10^{-5}$. Following Patterson et al. (1993), the mean variation in the superhump period in SU UMa superhumping sytems is $|\dot{P}| \simeq 3-10 \times 10^{-5}$ (see their Table. 1). For the recurrent nova VY Aqr, the authors derived a variation of $|\dot{P}| \simeq 8.2 \times 10^{-5}$. Our value for $|\dot{P}|$ probably comes from the errors in the period measurements rather than from real period changes. The period then seems to be constant during our observations, and the shape of the folded light curve suggests a primary and a secondary eclipse. We therefore propose that this periodicity is the orbital period of the binary system. Such a period is at the lower end of the mostly populated region of orbital periods in novae (Warner 2002). The dominant first harmonic frequency in the power spectra is a result of the clear structure primary - secondary eclipse in the folded light curve, which suggests a high inclination angle of the binary system. 
Using the orbital period of the system and Eq. (9) from Smith $\&$ Dhillon (1998), we obtain a rough estimate for the secondary star mass of $0.26 \pm 0.05 M_{\odot}$. Using a mean white-dwarf mass of $0.85 \pm 0.05 M_{\odot}$ from Smith \& Dhillon (1998), we find a mass ratio (secondary/primary mass) of $M_{2} / M_{1}=0.3 \pm 0.1$.

After the nova explosion, the hot white dwarf may heat up and irradiate the cooler companion. The observed orbital light curve of the nova can be the result of the aspect variations or eclipses of the secondary due to heating from the hot primary, and the asymmetry in the pulse profiles could be produced once the shape of the secondary approaches that of a tear drop model. The irradiation effects in classical novae can also be detected long after the outburst stage (e.g., V1500 Cyg; Sommers \& Naylor 1999, DN Gem; Retter et al. 1999). Two eclipse-like features are present in the folded light curve of V5116 Sgr (Fig. 6). The shape is similar to the light curve of V2540 Oph in 2003 (Ak et al. 2005) with a dip at phase $\sim 0.5(\sim 33 \%$ amplitude of the primary minimum for V2540 Oph and $\sim 70 \%$ for V5116 Sgr). The authors conclude that V2540 Oph is likely a high inclination system either showing an irradiation effect or having a spiral structure in its accretion disc. Woudt \& Warner (2003a) note that one of the following requirements must be fulfilled for the large-amplitude orbital modulations to be seen in the light curve of a recent nova in which the accretion disc does not dominate the luminosity of the system: 1) the disc is foreshortened but the secondary is seen (a high inclination angle), 2) the disc has small dimensions, 3 ) there is no disc (case of polars). In the case of V5116 Sgr, the polar interpretation is possible because of the orbital period distribution of polars that peaks below $5 \mathrm{~h}$ (Warner 1995), but nothing else indicates this option. The small dimension of the disc is supported by 1) the short orbital period, and 2) the post nova stage when the disc is reforming after explosion.

A comparison to two novae within the period gap IM Nor and DD Cir with present irradiation effect (Woudt \& Warner $2003 \mathrm{~b}, \mathrm{c})$ can be made. The light curve of V5116 Sgr is different from those of IM Nor and DD Cir but similar to V2540 Oph. IM Nor and DD Cir showed very small dips at phase 0.5 interpreted as partial eclipses of the irradiated secondary by the disc or matter stream. The light curve shape depends on the disc radius and on the inclination angle. The deep secondary eclipses in V5116 Sgr require a large disc or a high inclination. The strength of the irradiation effect (white dwarf post-outburst temperature) can also play a significant role. The differences in the phase of the maxima in Figs. $6 \mathrm{~b}$ and $\mathrm{c}$ can be due to spiral structures in the disc as mentioned before for the nova V2540 Oph.

The reconstruction of an accretion disc after the nova eruption is indicated by the discovery of the white dwarf spin or by the superhump period. A probable spin period of the white dwarf was detected $\sim 1$ yr after the outburst in V4745 Sgr (Dobrotka et al. 2006a), 2.75 yr in V4743 Sgr (Kang et al. 2006b), and $\sim 15$ months after the maximum in V1425 Aql (Retter et al. 1998). Several systems show superhumps as early as two and a half months after the outburst like V4633 Sgr (a spin period is another option in this case, Lipkin et al. 2001) or two years after the outburst like V1974 Cyg (Retter et al. 1997). In our V5116 Sgr light curves extending $\sim 470$ days after outburst, we did not find any photometric variations consistent with white dwarf spin modulation or superhump properties. We cannot say anything about the magnetic nature of the white dwarf, but the component's mass ratio indicates that the superhump existence is likely. The mass ratio using the primary mass average is not decisive. Using the derived secondary mass $0.26 M_{\odot}$ (Sect. 4.2), the mass ratio is lower than 0.35 for a primary mass higher than $0.74 M_{\odot}$. Taking all known primary masses in cataclysmic variables (Ritter \& Kolb 2003), 63\% have higher or equal mass than $0.74 M_{\odot}$. This probability value is not enough to make certain that superhumps are expected inV5116 Sgr, but the search for such variability could be fruitful; however V5116 Sgr is a very fast nova. According to Eq. (13) from Livio (1992) and taking $t_{3}=20.2 \pm 1.9 \mathrm{~d}$ derived in this paper (Sect. 3.1), we obtain a mass estimate of the white dwarf of $1.04 \pm 0.02 M_{\odot}$ and thus $M_{2} / M_{1}=0.25 \pm 0.05$. The presence of superhumps is then expected if a disc is fully developed up to tidal radius.

The superhump period is a few percent longer or shorter than the orbital period. The possibility that the periodicity found in this paper is a superhump is rejected following the stability discussion and the shape of the folded light curve (Fig. 6). The mean shape of superhumps is typically an asymetric sinusoid and our data show typical eclipse like features.

The presence of the disc in cataclysmic variables depends on the mass loss from the secondary. The material supplied from the secondary within the period gap depends on the strength of the magnetic braking driving the secondary out of thermal equilibrium (mass loss time scale shorter than the thermal time scale). The stronger the braking, the wider the gap and the higher the upper end of the period gap. If the magnetic braking is low enough, the mass loss time scale may never become shorter than the thermal time scale. In the case of novae, another condition is important. The nova explosion heats the secondary that leads to an enhanced mass transfer. Therefore the complete absence of accretion discs in novae within the period gap is not expected.

\subsection{Searching for superhumps in other nova remnants}

The orbital period $2.462 \mathrm{~h}$ of the mentioned IM Nor (Woudt \& Warner 2003b) yields a secondary mass estimate of $0.20 M_{\odot}$ following Smith \& Dhillon (1998). The decay time $t_{3} \simeq 50 \mathrm{~d}$ (Kato et al. 2002) yields a primary mass estimate of $\simeq 0.86 M_{\odot}$ (Livio 1992 ), giving a mass ratio $\simeq 0.23$. Woudt $\&$ Warner (2003b) conclude that they had not observed the superhump modulation. This conclusion with the mass ratio safely lower than 0.35 indicates that the existence of a fully developed disc is not probable. DD Cir (Woudt \& Warner 2003c) has a period of $2.339 \mathrm{~h}$ yielding a secondary mass estimate of $0.18 M_{\odot}$. The decay time $t_{2} \simeq 4.5 \mathrm{~d}$ (Liller 1999; no other estimates are available) places the object in the class of very fast novae (similar to V5116 Sgr). The decay time $t_{3} \simeq 10 \mathrm{~d}$ following the equation $t_{3} \simeq 2.75 t_{2}^{0.88}$ from Warner (1995) gives a rough white dwarf mass estimate of $\simeq 1.16 M_{\odot}$ (mass ratio $\left.<0.2\right)$. Woudt \& Warner (2003b) interpreted the deep eclipse as obscuration of the disc and did not detect any periodicity near the orbital period in the period analysis. Therefore, following the tidal instability model, it is again probable that the disc is not fully developed up to $3: 1$ resonance radius 3 years after the outburst; however, the authors argue that the disc radius is $47 \%$ of the orbital separation. Using the third Kepler law, we obtain a disc radius of $\sim 3.1 \times 10^{10} \mathrm{~cm}$. The disc in DD Cir is then large enough to reach the 3:1 resonance radius calculated from the Eq. (3.39) in Warner (1995); $r_{3: 1} \sim 3 \times 10^{10} \mathrm{~cm}$. The result marginally contrasts with the absence of superhumps. With the derived values for V5116 Sgr, we get $r_{3: 1} \simeq 3.5 \times 10^{10} \mathrm{~cm}$ as an upper limit of the disc radius, when considering the absence of superhumps into consideration. The boundaries of the eclipse in our light curve are not as clear as in the DD Cir case, therefore not suitable to estimate the disc radius.

Comparison to other systems follows the same way as in the case of IM Nor and DD Cir. We took component masses from the Ritter \& Kolb's catalogue (2003), and other masses we are 
Table 2. Selected nova systems.

\begin{tabular}{|c|c|c|c|c|c|c|c|}
\hline System & $\begin{array}{l}P_{\text {orb }} \\
{[\mathrm{h}]}\end{array}$ & $\begin{array}{c}M_{2} \\
{\left[M_{\odot}\right]}\end{array}$ & $\begin{array}{c}t_{3} \\
{[\mathrm{~d}]}\end{array}$ & $\begin{array}{c}M_{1} \\
{\left[M_{\odot}\right]}\end{array}$ & $q$ & $\overline{\mathrm{SH}}$ & Reference \\
\hline RW UMi & 1.418 & 0.07 & 140 & 0.64 & 0.11 & $\overline{\mathrm{N}}$ & 1 \\
\hline CP Pup & 1.474 & 0.08 & 8 & 1.19 & 0.07 & Y & 2 \\
\hline V1974 Cyg & 1.950 & 0.14 & 42 & 0.90 & 0.16 & Y & 3,4 \\
\hline RS Car & 1.980 & 0.14 & (58) & 0.83 & 0.17 & $\mathrm{~N}$ & 5 \\
\hline DD Cir & 2.339 & 0.18 & (10) & 1.16 & 0.16 & $\mathrm{~N}$ & 6 \\
\hline IM Nor & 2.462 & 0.20 & 50 & 0.86 & 0.23 & $\mathrm{~N}$ & 7,8 \\
\hline QU Vul & 2.682 & 0.23 & 40 & 0.91 & 0.25 & $\mathrm{~N}$ & 9 \\
\hline V2214 Oph & 2.804 & 0.24 & 100 & 0.71 & 0.34 & $\mathrm{~N}$ & 10 \\
\hline V630 Sgr & 2.830 & 0.25 & 11 & 1.14 & 0.22 & Y & 11 \\
\hline V351 Pup & 2.837 & 0.25 & (32) & 0.95 & 0.26 & $\mathrm{~N}$ & 11 \\
\hline V5116 Sgr & 2.971 & 0.26 & 20.2 & 1.04 & 0.25 & $\mathrm{~N}$ & this paper \\
\hline V4633 Sgr & 3.014 & 0.27 & 42 & 0.90 & 0.30 & Y & 12 \\
\hline DN Gem & 3.068 & 0.28 & 37 & 0.92 & 0.30 & $\mathrm{~N}$ & 13 \\
\hline V1494 Aql & 3.231 & 0.30 & 16 & 1.08 & 0.28 & $\mathrm{~N}$ & $14,15,16$ \\
\hline V1668 Cyg & 3.322 & 0.31 & 23 & 1.02 & 0.30 & $\mathrm{~N}$ & 17 \\
\hline V603 Aql & 3.324 & $0.31(0.29)$ & 8 & $1.19(1.2)$ & $0.26(0.24)$ & Y & 18 \\
\hline RR Cha & 3.370 & 0.31 & 58 & 0.83 & 0.37 & Y & 5,19 \\
\hline RR Pic & 3.481 & $0.33(0.4)$ & 150 & $0.62(0.95)$ & $0.53(0.42)$ & $\mathrm{N}$ & 20,21 \\
\hline V382 Vel & 3.508 & 0.33 & 9 & 1.17 & 0.28 & $\mathrm{~N}$ & 22,23 \\
\hline V533 Her & 3.530 & 0.33 & 44 & 0.89 & 0.37 & $\mathrm{~N}$ & 24,25 \\
\hline V2574 Oph & 3.550 & 0.34 & (33) & 0.95 & 0.36 & Y & 26 \\
\hline OY Ara & 3.731 & $0.36(0.34)$ & . & $(0.82)$ & $(0.41)$ & $\mathrm{N}$ & 27 \\
\hline V1493 Aql & 3.740 & 0.36 & 7 & 1.21 & 0.30 & $\mathrm{~N}$ & 14,28 \\
\hline V849 Oph & 4.146 & 0.41 & 175 & 0.59 & 0.69 & $\mathrm{~N}$ & 29 \\
\hline DQ Her & 4.647 & $0.48(0.40)$ & 94 & $0.72(0.60)$ & $0.67(0.67)$ & $\mathrm{N}$ & 30 \\
\hline $\mathrm{T}$ Aur & 4.906 & $0.51(0.63)$ & 100 & $0.71(0.68)$ & $0.72(0.92)$ & $\mathrm{N}$ & 31 \\
\hline V4745 Sgr & 4.987 & 0.52 & 32.8 & 0.95 & 0.55 & $\mathrm{~N}$ & 32,33 \\
\hline HR Del & 5.140 & $0.54(0.55)$ & 230 & $0.54(0.67)$ & $1.00(0.82)$ & $\mathrm{N}$ & 34 \\
\hline V1425 Aql & 6.139 & 0.57 & 39 & 0.91 & 0.63 & $\mathrm{~N}$ & 14,35 \\
\hline V4743 Sgr & 6.718 & 0.74 & 15 & 1.10 & 0.67 & $\mathrm{~N}$ & 36,37 \\
\hline BY Cir & 6.760 & 0.74 & 157 & 0.62 & 1.19 & $\mathrm{~N}$ & 6 \\
\hline V2540 Oph & 6.835 & 0.75 & 213 & 0.55 & 1.36 & $\mathrm{~N}$ & 38 \\
\hline V838 Her & 7.143 & $0.79(0.74)$ & 3.2 & $1.29(0.87)$ & $0.61(0.85)$ & $\mathrm{N}$ & 39 \\
\hline V2275 Cyg & 7.548 & 0.84 & 7 & 1.21 & 0.69 & $\mathrm{~N}$ & 40,41 \\
\hline BT Mon & 8.012 & $0.90(0.87)$ & (213) & $0.55(1.04)$ & $1.64(0.84)$ & $\mathrm{N}$ & 42 \\
\hline V368 Aql & 8.285 & 0.93 & 42 & 0.90 & 1.03 & $\mathrm{~N}$ & 43 \\
\hline QZ Aur & 8.580 & $0.97(1.05)$ & 27 & $0.99(1.05)$ & $0.98(1.00)$ & $\mathrm{N}$ & 44 \\
\hline
\end{tabular}

Note: $P_{\text {orb }}$ is the orbital period in hours, $M_{2}$ is the secondary mass in solar masses derived from Smith \& Dhillon (1998) (from Ritter \& Kolb 2003 , in parentheses), $t_{3}$ is the decline time of three magnitudes from maximum (value in parentheses is the value estimated from the $t_{2}$ time using $t_{3} \simeq 2.75 t_{2}^{0.88}$ from Warner 1995), $M_{1}$ is the primary mass derived from Livio (1992) (from Ritter \& Kolb 2003, in parentheses), $q$ is the mass ratio, $\mathrm{SH}$ is the information about superhump detection ( $\mathrm{Y}-$ yes, $\mathrm{N}-$ no), and Ref. is the reference.

References: 1 - Retter \& Lipkin (2001), 2 - Patterson \& Warner (1998), 3 - Skillman et al. (1997), 4 - Retter et al. (1997), 5 - Woudt \& Warner (2002), 6 - Woudt \& Warner (2003c), 7 - Woudt \& Warner (2003b), 8 - Kato et al. (2002a), 9 - Shafter \& Misselt (1995), 10 - Baptista et al. (1993), 11 - Woudt \& Warner (2001), 12 - Lipkin et al. (2001), 13 - Retter et al. (1999), 14 - Arkhipova et al. (2002), 15 - Kato et al. (2004), 16 Barsukova \& Goranskii (2003), 17 - Kaluzny (1990), 18 - Retter \& Naylor (2000), 19 - Hoffmeister (1959), 20 - Vogt (1975), 21 - Warner (1986), 22 - Balman et al. (2006), 23 - Della Valle et al. (2002), 24 - Patterson (1979b), 25 - Thorstensen \& Cynthia (2000), 26 - Kang et al. (2006a), 27 Zhao \& McClintock (1997), 28 - Dobrotka et al. (2006b), 29 - Shafter et al. (1993), 30 - Walker (1961), 31 - Walker (1963), 32 - Csák et al. (2005), 33 - Dobrotka et al. (2006a), 34 - Kohoutek \& Pauls (1980), 35 - Retter \& Leibowitz (1998), 36 - Kang et al. (2006b), 37 - Kato et al. (2002b), 38 - Ak et al. (2005), 39 - Leibowitz et al. (1992), 40 - Balman et al. (2005), 41 - Kiss et al. (2002), 42 - Robinson et al. (1982), 43 - Diaz \& Bruch (1994), 44 - Campbell \& Shafter (1995).

Aditional sources for orbital period, $t_{2} / t_{3}$ time and catalogue masses: Duerbeck (1987), Warner (1986, 1995, 2002), Diaz \& Bruch (1997), Ritter \& Kolb (2003).

estimated from the orbital period (using Smith \& Dhillon 1998) and $t_{3}$ time (using Livio 1992). We rejected systems with unknown or non measurable $t_{2}$ or $t_{3}$ time (peculiar light curve, strong oscillations in the decline) without catalogue mass values (no information on mass ratio, ex: RS Car), magnetic novae (AM Her systems without disc, ex: V1500 Cyg), systems with orbital period $>10 \mathrm{~h}$ (Smith and Dhillon fitting not adequate, systems with evolved secondary are then excluded too, ex: DI Lac, V841 Oph, V723 Cas, GK Per), systems with insufficient photometric observations to detailed period analysis (ex: V500 Aql,
V446 Her, HZ Pup, DY Pup, CT Ser). The final list of systems is in Table 2.

The results are depicted in Fig. 7. The lower panel only shows the systems with known catalogue mass values and the upper panel covers the addition of estimated mass values in this work. The critical mass ratio of 0.35 is also shown. The results presented in the lower panel are as expected from the mass ratio limit for superhumps, but the statistical set is small. Adding other mass estimates changes the situation. Seven systems below or close to the limit 0.35 show superhumps but 14 systems do 


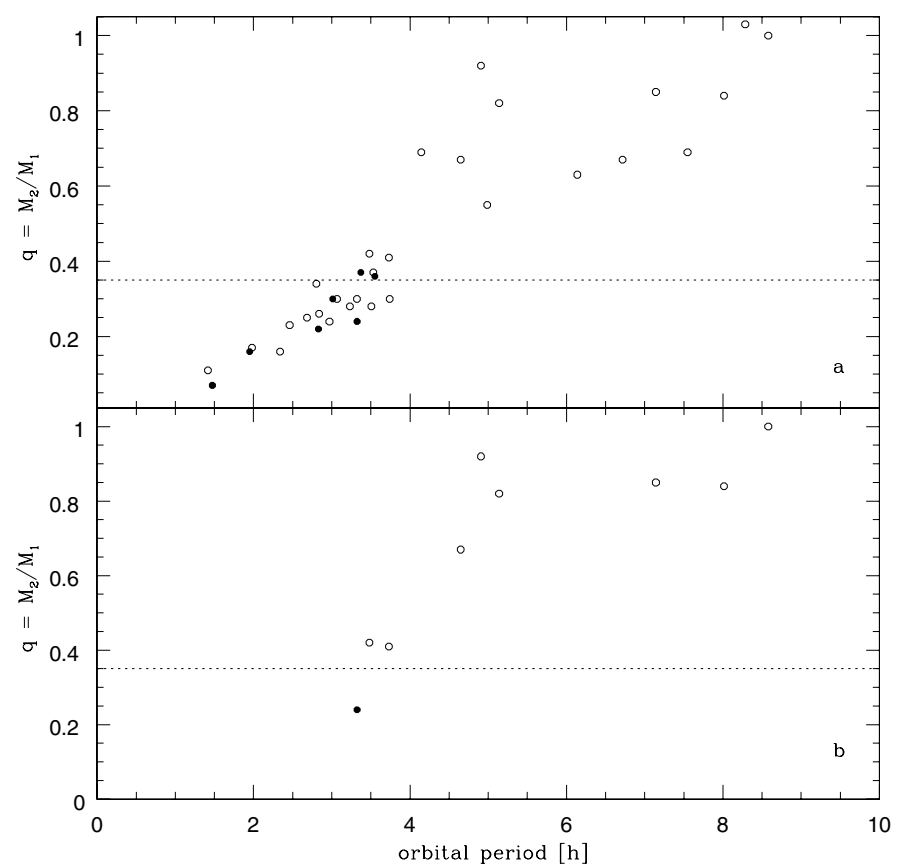

Fig. 7. Selected nova remnants in the mass ratio $(q)$ - orbital period $\left(P_{\text {orb }}\right)$ plane. Open circle - non detected superhumps; filled circle - detected superhumps. Panel a) - mass ratio from combined sources (catalogue and estimates from $P_{\text {orb }}, t_{3}$ time); panel b) - mass ratio only from catalogue values (Ritter \& Kolb 2003). The dashed line is the 0.35 limit.

not. The estimated mass ratio has a mean difference of 0.2 from the catalogue values. The differences are distributed randomly. Therefore there is no reason to suspect that all mass ratios are systematically underestimated. The seven systems with detected superhumps are safely located in comparing to the 0.35 critical value, as expected by theory, and are only a third of all systems falling below or close to the mass ratio of 0.35 . The majority of all systems occupy the orbital period interval of $2-4 \mathrm{~h}$. Following Patterson (2005), this interval has a $\sim 40-90 \%$ probability of observing superhumps. Seven systems versus 14 from our investigation yields only $\sim 30 \%$.

Superhumps were observed during the outbursts of short orbital period dwarf novae (SU UMa stars) where the disc reaches the 3:1 resonance radius (superoutburst case). The disc during this active stage (outburst - active and hot stage, quiescence - not active and cold stage) reaches larger radius than in quiescence (see Lasota 2001, for review). The irradiation of the secondary by the central white dwarf in nova remnants can produce an enhanced mass transfer rate that is strong enough to keep the disc in this stable hot active stage (as in the nova like stars) with the larger diameter (Hameury \& Lasota 2005). In addition, superoutbursts in SU UMa stars, when superhumps are observed, can be explained by the irradiation and the enhanced mass transfer (Smak 1995, 2004; Hameury et al. 2000; Schreiber et al. 2004). Therefore, permanent superhumpers are possible after a nova outburst. Some of the possible interpretations of the superhump lack can be: 1) not systematically applicable tidal instability theory (tidal torques are probably not the main and only condition of superhump existence as concluded by Hameury \& Lasota 2005), 2) disc radius not developed up to the tidal radius, 3) superhumps present but not detected because of insufficient sets of data.

\section{Summary}

By using the maximum visual magnitude of $V=7.15$, we measured the decay time $t_{2}=6.5 \pm 1.0 \mathrm{~d}$ from the long-term light curve. This makes V5116 Sgr a very fast nova. We estimated a visual absolute magnitude in maximum of $M_{\mathrm{V} \max }=$ $-8.85 \pm 0.04 \mathrm{mag}$. We found a period of $0.1238 \pm 0.0001 \mathrm{~d}$ $(2.9712 \pm 0.0024 \mathrm{~h})$, which we interpret as the orbital period of the binary system. We propose that V5116 Sgr is a high inclination system showing a strong irradiation effect of the secondary star. No fully developed accretion disc up to the tidal radius is possible with a radius lower than $3.5 \times 10^{10} \mathrm{~cm}$.

Searching for superhumps in nova remnants using the limiting value for mass ratio 0.35 and estimated mass values shows that, in the majority of systems with expected superhumps, this variability has not been found yet. Looking for superhumps in these systems could be fruitful.

Acknowledgements. This work was supported by the Slovak Academy of Sciences Grant No. 2/7011/7. We acknowledge with thanks the variable star observations from the AAVSO International Database contributed by observers worldwide and used in this research. We also acknowledge Michael Friedjung for his valuable comments.

\section{References}

Ak, T., Retter, A., \& Liu, A. 2005, PASA, 22, 298

Arkhipova, V. P., Burlak, M. A., \& Esipov, V. F. 2002, Astron. Lett., 28, 100

Balman, S., Yilmaz, A., Retter, A., Saygac, T., \& Esenoglu, H. 2005, MNRAS, 356,773

Balman, S., Retter, A., \& Bos, M. 2006, AJ, 131, 2628

Baptista, R., Jablonski, F. J., Cieslinski, D., \& Steiner, J. E. 1993, ApJ, 406, L67 Barsukova, E. A., \& Goranskii, V. P. 2003, Astron. Lett., 29, 195

Csák, B., Kiss, L. L., Retter, A., Jacob, A., \& Kaspi, S. 2005, A\&A, 429, 599

Campbell, R. D., \& Shafter, A. W. 1995, ApJ, 440, 336

Della Valle, M., \& Livio, M. 1995, ApJ, 452, 704

Della Valle, M., Pasquini, L., Daou, D., \& Williams, R. E. 2002, A\&A, 390, 155

Diaz, M. P., \& Bruch, A. 1994, IBVS, 4079, 1

Diaz, M. P., \& Bruch, A. 1997, A\&A, 322, 807

Dobrotka, A., Retter, A., \& Liu, A. 2006a, MNRAS, 371, 459

Dobrotka, A., Friedjung, M., Retter, A., Hric, L., \& Novák, R. 2006b, A\&A, 448,1107

Drake, J. J., Wagner, R. M., Starrfield, S., et al. 2003, ApJ, 584, 448

Duerbeck, H. W. 1987, Sp. Sci. Rev., 45, 1

Gilmore, A. C., \& Kilmartin, P. M. 2005, IAUC, 8559

Hameury, J.-M., \& Lasota, J.-P. 2005, A\&A, 443, 283

Hameury, J.-M., Lasota, J.-P., \& Warner, B. 2000, A\&A, 353, 244

Hellier, C. 1996, in Cataclismic Variable and Related Objects, ed. N. Evans, \& J. H. Wood (Dordrecht: Kluwer), IAU Coll., 158, 143

Hoffmeister, C. 1959, AN, 285, 149

Jacques, C. 2005, IAUC, 8559

Kaluzny, J. 1990, MNRAS, 245, 547

Kang, T. W., Retter, A., Liu, A., \& Richards, M. 2006a, AJ, 131, 1687

Kang, T. W., Retter, A., Liu, A., \& Richards, M. 2006b, AJ, 132, 608

Kato, T., Yamaoka, H., Liller, W., \& Monard, B. 2002a, A\&A, 391, 7

Kato, T., Hishikura, T., West, J. D., et al. 2002b, IAUC, 7976

Kato, T., Ishioka, R., Uemura, M., Starkey, D., \& Krajci, T. 2004, PASJ, 56, 125

Kiss, L. L., Gogh, N., Vinkó, et al. 2002, A\&A, 384, 982

Kohoutek, L., \& Pauls, R. 1980, A\&A, 92, 200

Lasota, J.-P. 2001, New Asron. Rev., 45, 449

Leibowitz, E. M., Mendelson, H., \& Mashal, E. 1992, ApJ, 385, L49

Lipkin, Y., Leibowitz, E. M., Retter, A., \& Shemmer, O. 2001, MNRAS, 328, 1169

Liller, W. 2005, IAUC, 8559

Livio, M. 1992, ApJ, 393, 516

Ness, J. U., Starrfield, S., Burwitz, V., et al. 2003, ApJ, 594, L127

Paczyński, B. 1971, Acta Astron., 21, 417

Patterson, J. 1978, ApJ, 225, 954

Patterson, J. 1979a, ApJ, 231, 789

Patterson, J. 1979b, ApJ, 233, L13

Patterson, J. 1994, PASP, 106, 697

Patterson, J., \& Warner, B. 1998, PASP, 110, 1026

Patterson, J., Bond, H. E., Grauer, A. D., Shafter, A. W., \& Mattei, J. A. 1993, PASP, 105, 69 
Patterson, J., Kemp, J., Harvey, D. A., et al. 2005, PASP, 117, 1204

Pringle, J. E. 1981, Ann. Rev. Astron., 19, 137

Retter, A. 2002, in Classical Nova Explosion, ed. Hernanz, M., \& José, J., AIP Conf. Ser. 637, 279

Retter, A., \& Leibowitz, E. M. 1998, MNRAS, 296, L37

Retter, A., \& Lipkin, Y. 2001, A\&A, 365, 508

Retter, A., \& Naylor, T. 2000, MNRAS, 319, 510

Retter, A., Leibowitz, E. M., \& Ofek, E. O. 1997, MNRAS, 286, 745

Retter, A., Leibowitz, E. M., \& Kovo-Kariti, O. 1998, MNRAS, 293, 145

Retter, A., Leibowitz, E. M., \& Naylor, T. 1999, MNRAS, 308, 140

Ritter, H., \& Kolb, U. 2003, A\&A, 404, 301

Robinson, E. L., Nather, R. E., \& Kepler, S. O. 1982, ApJ, 254, 646

Russell, R. W., Lynch, D. K., \& Rudy, R. J. 2005, IAUC, 8579

Schmidt, G. D., \& Stockman, H. S. 1991, ApJ, 371, 749

Schreiber, M. R., Hameury, J.-M., \& Lasota, J.-P. 2004, A\&A, 427, 621

Shafter, A. W., \& Misselt, K. A. 1995, ApJ, 448, L33

Shafter, A. W. Misselt, K. A., \& Veal, J. M. 1993, PASP, 105, 853

Skillman, D. R., Harvey, D., Patterson, J., \& Vanmunster, T. 1997, PASP 109, 114

Sommers, M. W., \& Naylor, T. 1999, A\&A, 352, 563

Smak, J. 1995, AcA, 45, 355
Smak, J. 2004, AcA, 54, 221

Smith, D. A., \& Dillon, V. S. 1998, MNRAS, 301, 767

Stellingwerf, R. F. 1978, ApJ, 224, 953

Thorstensen, J. R., \& Cynthia, J. T. 2000, MNRAS, 312, 629

Vogt, N. 1975, A\&A, 41, 15

Walker, M. F. 1961, ApJ, 134, 171

Walker, M. F. 1963, ApJ, 138, 313

Warner, B. 1986, MNRAS, 219,75

Warner, B. 1986, MNRAS, 222, 11

Warner, B. 1995, Cataclysmic Variable Stars (Cambridge: Cambridge University Press)

Warner, B. 2002, in Classical Nova Explosions, ed. M. Hernanz, \& J. José, AIP Conf. Ser., 637, 3

Webbink, R. F. 1990, in Accretion-Powered Compact Binaries, ed. C. W. Mauche (Cambridge: Cambridge University Press), 177

Woudt, P. A., \& Warner, B. 2001, MNRAS, 328, 159

Woudt, P. A., \& Warner, B. 2002, MNRAS, 335, 44

Woudt, P. A., \& Warner, B. 2003a, MNRAS, 339, 731

Woudt, P. A., \& Warner, B. 2003b, MNRAS, 343, 313

Woudt, P. A., \& Warner, B. 2003c, MNRAS, 340, 1011

Zhao, P., \& McClintock 1997, ApJ, 483, 899 